\title{
Development and implementation of a green logistics-oriented framework for batch process industries: two case studies
}

\author{
Nagham M. El-Berishy ${ }^{1}$ (D) Bernd Scholz-Reiter ${ }^{1}$
}

Received: 17 December 2015/Accepted: 3 June 2016/Published online: 15 June 2016

(c) The Author(s) 2016. This article is published with open access at Springerlink.com

\begin{abstract}
In recent years, green logistics has received increasing interest due to the rise in greenhouse gases emission from transport operations. It is a promising approach to managing supply chain decisions to reduce environmental damages. Many models have been developed in the literature to study the environmental aspects of routing problems. However, these models have not been implemented in practice, particularly in batch processing industries. In this paper, we developed a two-stage stochastic model which integrates the tactical and operational decision levels to reduce the economic and environmental impacts of transport activities. This model generates optimum vehicle routes and their delivery velocities with the objective of minimizing the total distribution cost and the related emissions. We implemented the proposed model in two case studies drawn from the fast-moving consumer goods industry. This resulted in a cost reduction of up to $13 \%$ of the total related economic and environmental costs compared to the actual situation at the test sites. These results also show the potential of green logistics-based models to improve the current modelling capabilities for batch distribution planning.
\end{abstract}

This article is part of a focus collection on "Dynamics in Logistics: Digital Technologies and Related Management Methods".

Nagham M. El-Berishy

elb@biba.uni-bremen.de

Bernd Scholz-Reiter

bsr@biba.uni-bremen.de

1 International Graduate School (IGS) for Dynamics in Logistics, University of Bremen, 28359 Bremen, Germany
Keywords Green logistics - Batch process industry · Supply chain distribution - Carbon emissions . Environmental objective

\section{Introduction}

The ever-growing population worldwide and their consumption of basic need goods (such as fast-moving consumer goods, beverages, cleaning products) pose new challenges for research in distribution planning. These goods are commonly produced in batches due to their low unit volume. In order to meet the requirements for high consumption rate, optimum distribution plans for batch products have to be generated across different locations. An effective and efficient management of batch flow leads to huge savings in absolute terms for both the companies and the consumers [10]. Additionally, the growth in road traffic and the use of road freight transport raise the level of emitted greenhouse gases (GHG) in the environment. As one of today's top market concerns, all aspects of an organization's logistics must be green [15]. This shift towards greening the supply chain through an environmentally friendly logistics network design is not only to abide by governmental regulations but also to meet customers' expectations and social responsibilities [22].

Distribution planning can support decision making by generating the optimum routes, minimizing the total transportation costs as well as saving energy and, consequently, GHG emissions. The optimum delivery routes for each vehicle are generated at the tactical level (route planning) and the optimum delivery velocities at the operational level (schedule planning). Therefore, the model described in this paper is formulated as two-stage stochastic model. The model integrates the decisions made 
at the two decision levels to reduce the environmental impact of transport activities. Also, GHG emissions under uncertainties are incorporated as a function of randomly selected delivery velocities at different distribution routes within the supply network. The vehicle velocity has the most significant effects on fuel consumption [20]. Thus, optimal speed could lead to greater reduction of $\mathrm{CO}_{2}$ emissions and fuel consumption for a given routing plan [6].

Although there are a number logistics models in the literature, there is a lack of research into real-life applications underpinned by case studies [23]. The current study validates a newly developed green logistics model via two real-world case studies of multinational organizations drawn from the batch process industry.

This paper is organized as follows: Sect. 2 reviews the extant literature on implementation of green logistics models in batch distribution. This section also describes the case studies and the background to the study. Section 3 defines the methodology and the development of optimum distribution routes, optimum delivery velocities and related $\mathrm{CO}_{2}$ emissions. Computational results and discussions are presented in Sect. 4. Finally, the conclusions and main findings are highlighted in Sect. 5.

\section{Greening batch distribution: literature review}

There has been a marked increase in the consumption of goods produced using batch processes. This has resulted in a commensurate rise in production and related distribution operations [21]. Consequently, these related distribution operations have contributed immensely to the total global transport emissions. According to Buehler and Pucher [2], a fifth of worldwide GHG emissions are due to transport activities. In fact, the transportation sector will be the second largest emitter accounting for $24 \%$ of the total EU emissions in 2020. Therefore, there is a renewed sense of urgency to investigate the environmental parameters during transport operations and develop appropriate distribution planning models [12]. Scheduling most of the distribution plans is based on the vehicle routing problem (VRP) [17]. The VRP is a classical combinatorial optimization problem that has become a key component of distribution and logistics management. Through VRPs, the reduction in total vehicle's travelled distance, by itself, provides environmental benefits. This is due to the reduction in fuel consumption, the consequent drop in pollutants and resultant shorter lead times [3, 5, 7].

In recent research contributions, there have been a number of models suggested to minimize the emissions of GHG associated with vehicle routing problems based on vehicle velocities. The velocity of vehicles is the major determinant of environmental emissions for a specific vehicle load [6]. The usage of speed to minimize emissions in VRP models can be classified into two main categories: the first is the set of models where vehicle speeds are inputs to the model and the second consists of models where vehicle speed values are optimized and used as decision variables.

Furthermore, Kumar et al. [13] pointed out the necessity of implementing integrated optimization models that combine operational and environmental costs elements in real case studies. Moreover, implementing these models in real environment plays a vital role in exploring their validity in achieving environmentally sustainable logistics practices [9]. As an application of green logistics concept in the batch process industry, Ubeda et al. [23] examined aspects of green transportation at the operational level where vehicles speeds are inputs and proposed some green changes within the VRP based on a case study at one of the leading distribution companies in Spain. They found that an average saving of $25.5 \%$ in $\mathrm{CO}_{2}$ emissions was possible due to reduction in used routes compared to the prevailing situation in that company at the time.

Moreover, Pishvaee et al. [19] proposed a bi-objective fuzzy mathematical programming model for green logistics design under uncertain conditions. Their model integrated the transportation and production strategic decisions, and its applicability was examined in an industrial case study. As their decisions were at the strategic level, velocity speeds were reported as inputs as well. In contrast, Maden et al. [14], using vehicle speed as decision variables, minimized the total vehicle emissions per unit distance required for distribution operations. $\mathrm{A} \mathrm{CO}_{2}$ saving of around $7 \%$ was recorded using a tabu search algorithm. Similarly, Qian and Eglese [20] minimized the fuel emissions in a road network where speed of each vehicle along each road in its path is treated as decision variables.

The integrated model presented in this paper is novel given that velocity values were used both as inputs and decision variables in an optimization model. A two-stage stochastic model is designed to generate the global optimum solution for both tactical and operational decision levels. In the first stage, the vehicle velocities are known only within certain bounds and considered as inputs to the model. The optimum values for these velocities are uncertain at this stage. Once the optimum routes are known, the delivery velocities are used as decision variables in the second stage. The optimum delivery velocities which correspond to the minimum emission values are then generated. Integrating emission values within the modelling of distribution plans is essential in order to achieve a sustainable balance between economic and environmental objectives [16]. 
As the implementation of the developed model in case studies is as crucial as developing these approaches and models, data were collected from two real-world case studies of multinational batch process companies. In each case study, data used for the numerical analysis belong to one full month of activities. The data showed a regular trend.

In the next sections, we will focus on generating the optimal vehicle routing that would enable reduction of the environmental impact by optimizing the distribution planning. Thereafter, we will propose a practical framework which considers both tactical and operational decisions. We will also discuss the implementation of this framework using the two case studies.

\section{Methodology and case studies}

In this section, the methodology used to handle batch distributions is described. This section composes two subsections: defining the environmental values and developing the mathematical model. Afterwards, the model is implemented in two case studies from batch process industry in order to verify the applicability of the model in practice. The characteristics of the test problems and some implementation details are described. The optimal delivery routes sequencing, the corresponding numbers of required delivery trucks and the optimum delivery speeds required to achieve the environmental targets were then determined.

\subsection{Environmental values}

Although there is no standard way to measure the values of fuel consumption and GHG emissions for a mobile source, a number of formulas could be applied to easily calculate their approximate values. Generally, there are two ways to calculate $\mathrm{CO}_{2}$ emissions: fuel-based or distance-based methodology [23]. While the fuel-based method is more reliable, the distance-based method is more popular due to the type of the data required. The distance-based method of estimating $\mathrm{CO}_{2}$ emissions will be used in this article due to readily available data. Two recorded values are required to calculate emissions based on this method: the distance travelled $\left(D_{l k}\right)$ and fuel consumption $\left(\mathrm{EM}_{l k}\right)$ by vehicles [23]. Additionally, this research used values of velocities instead of distances.

Delivery velocities are defined as vectors of uncertain values on each route (between any two nodes in the network) in each period. This vector can be mathematically formulated as follows: $\Omega=(\operatorname{Min} V, \operatorname{Max} V)$ where $\operatorname{Min} V$ and $\operatorname{Max} V$ correspond to the minimum and maximum vehicle velocity. It is expected that each vehicle is driven with velocity $\left(V_{t l k}\right)$ and results in amount of emissions $\left(E_{t l k}\right)$. A range of $\operatorname{Min} E$ and $\operatorname{Max} E$ corresponds to a range of delivery vehicle velocities $\operatorname{Max} V_{t l k}(\omega)$ and $\operatorname{Min} V_{t l k}(\omega)$ where $\omega$ is a given value of uncertain parameters. If these values are not practically applicable, penalties for overage emissions $F_{l k}$ (paid to the governments) and that for underage emissions $G_{l k}$ (associated with delivery delays for customers) are imposed. The idea behind this definition of penalties is to obtain meaningful combinations of companies and customers concerns. From the companies' perspective, the goal is to deliver the batches to different customers as efficiently as possible (concerning economic cost and overage emissions which are paid to the governments). From the customers' point of view, the main concern is to reliably receive the deliveries on time. Therefore, the objective function has emissions limits and stochastic travel velocities, leading to stochastic arrival times.

\subsection{The mathematical formulation}

The proposed study presents a research methodology with a mathematical core based on both the economic and environmental data as well as the capacity constraints. Six-step procedure was applied to realize the proposed solution methodology as shown in Fig. 1. The first step is to build a model which captures the properties of the production process and its corresponding flows of materials. The second step is to extract all the required data in order to generate feasible production plans at minimum costs. Afterwards, the decision-maker at the plant uses this data as well as further knowledge or expectations about the current and future situation on the shop floor to generate a set of assumptions. These inputs are called scenarios. Then, an initial solution is generated for each given scenario. The fifth step is to analyse the production schedule and interactive modifications of the developed model based on the experience and knowledge of the decision-maker and the feasibility of the production plans. The sixth and last step in this procedure is to approve the generated solution. This approval is based on the decision-maker evaluation for all available alternatives. In reality, these steps should be followed by execution and updating.

In order to fulfil the demand of customers in $L$ locations, there are several products $P$ which are produced and grouped into batches during $T$ time periods. The distribution problem is modelled on a full graph $G=(V, L)$, where $V=\{1,2, \ldots, n\}$ as the set of vertices, and $L=\{(l, k): 1$, $k \in L, l \neq k\}$ as the set of arcs. The formulated problem exhibits the following characteristics: (a) known homogeneous fleet size (each vehicle has a capacity indicated by CAP), (b) single depot (denoted as $\mathrm{V}_{\mathrm{o}}$ ), (c) deterministic demand (each customer has a certain demand $A_{t l p}$ which should be delivered by their due dates $\mathrm{DD}_{p}$ ), (d) oriented 


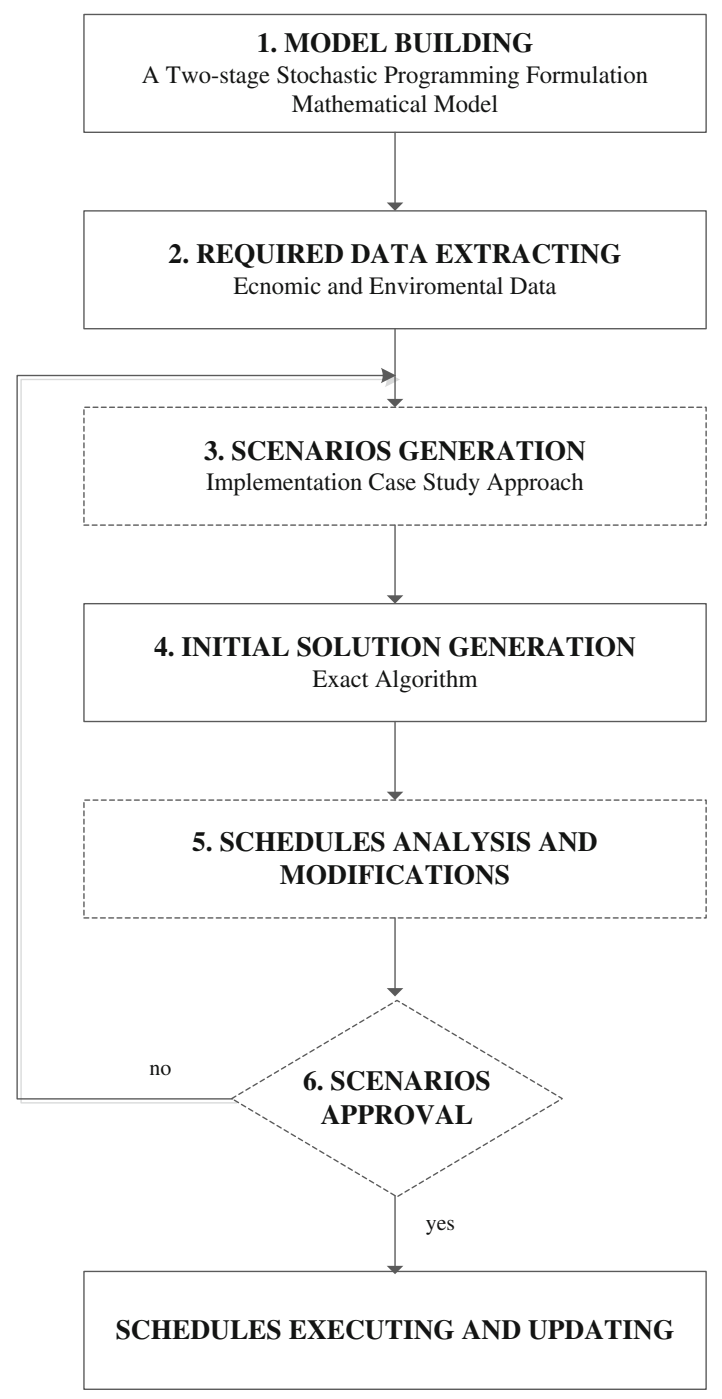

Fig. 1 Research methodology

network ( $n$ nodes represent geographically customer locations $L$ separated by distance $D_{l k}$ ), (e) the transportation cost from the depot to these locations can vary (a unit transportation cost $\mathrm{DC}_{l k}$ is a function of the vehicle travelled distance for delivering the customer orders) and (f) objective: minimizing total distribution costs.

Given this uncertainty, the two-stage stochastic programming model is developed because the model deals with two levels of decisions. Both stages present the tactical and operational decisions. The uncertainties are mostly found in the operational stage since most operational parameters are not fully known when the tactical decisions have to be made. The scenario-based approach is used in this paper to model the problem under uncertainty. The compact form of the stochastic model can be stated as follows $[1,18]$ :

$\min z=c^{T} x+E_{\xi}\left[\min q(\omega)^{T} y(\omega)\right]$ s.t. $\quad A x=b$

$T(\omega) x+W y(\omega)=h(\omega)$

$x \geq 0, \quad y(\omega) \geq 0$

The objective function contains a deterministic term $c^{T} x$ and the expectation of the second-stage objective $q(\omega)^{T} y(\omega)$ taken over all realizations of the random event $\omega$. The first-stage decisions are represented by the vector $x$ and matrices $c, b$ and $A$. In the second stage, a number of random events $\omega \in \Omega(\Omega$ denotes the set of all possible scenarios and $\omega$ a particular scenario) may be realized. For each $\omega$, the value $y(\omega)$ is the solution of a linear programme. For a given realization $\omega$, the second-stage problem data $q(\omega), h(\omega)$, and $T(\omega)$ become known. Each component of $q, h$, and $T$ is thus a possible random variable.

In the developed stochastic optimization model, the delivery velocities and their corresponding greenhouse gas emissions are considered as uncertain parameters. The second-stage decision variables are $E_{t l k}(\omega)$ the total carbon emissions value in route between locations $l$ and $k$ in time period $t$. Total produced carbon emissions over $\left(\mathrm{OE}_{t l k}(\omega)\right)$ and under $\left(\mathrm{UE}_{t l k}(\omega)\right)$ are the permissible limits in route between locations $l$ and $k$ in time period $t ; E_{t l k}(\omega)$ are defined. Additionally, we define binary decision variables $Z_{t l k}(l \neq k)$, equal to 1 if and only if the route $f$ location $l$ to location $k$ will be used in time period $t$ and 0 otherwise. Given the previous problem definition, the resulting formulation is given in the following steps by (5)-(20).

$$
\begin{aligned}
& \min \sum_{t \in T} \sum_{p \in P} \mathrm{DC}_{l k} \cdot D_{l k} \cdot Z_{t l k}+E_{\omega}[Q(X, H, Z, \omega)] \\
& \sum_{l \in L} Z_{t l k}=1 \quad \forall k \in L \backslash\{1, l\}, \quad t \in T
\end{aligned}
$$

$\sum_{k \in L} Z_{t l k}=1 \quad \forall l \in L \backslash\{1, k\}, \quad \forall t \in T$

$\sum_{p \in P} A_{t k p} \geq \sum_{p \in P} A_{t l p}-\mathrm{CAP}+\mathrm{CAP} \cdot\left(Z_{t k l}+Z_{t k}\right) \quad \forall t \in T, \quad \forall k \in L$

$$
\begin{aligned}
& \sum_{p \in P} A_{t k p} \leq \mathrm{CAP}-\left(\mathrm{CAP}-\sum_{p \in P} Q_{t k p}\right) \cdot Z_{t 1 k} \quad \forall t \in T, \quad \forall k \in L \\
& \sum_{p \in P} A_{t l p}-\sum_{p \in P} Q_{t l p}-\sum_{p \in P} \sum_{i \in L \backslash\{1\}} Q_{t i p} \cdot Z_{t i l} \geq 0 \quad \forall t \in T, \quad \forall l \in L
\end{aligned}
$$

$\sum_{p \in P} A_{t l p} \leq \mathrm{CAP} \quad \forall l \in L, t \in T$

$\sum_{k \in L \backslash\{1\}} Z_{t 1 k}-0.99 \geq \sum_{l \in L \backslash\{1\}} \frac{Q_{t l p}}{\mathrm{CAP}} \quad \forall t \in T, \quad \forall p \in P$ 
$Z_{t l k} \in\{0,1\} \quad \forall l \in L$

$A_{t l p} \geq 0 \quad \forall l \in L, \quad \forall t \in T, \quad \forall p \in P$

where $Q(X, H, Z, \omega)$ is the optimal value of the following second-stage problem:

$$
\begin{aligned}
& \operatorname{Min} Q(X, H, Z, \omega) \\
& =\sum_{l \in L} \sum_{k \in L \backslash\{l\}} \sum_{t \in T}\left[\left(\mathrm{EM}_{l k} \cdot B_{l}\right) \cdot V_{t l k}(\omega)+F_{l k} \cdot \mathrm{OE}_{t k}(\omega)+G_{l k} \cdot \mathrm{UE}_{t k}(\omega)\right]
\end{aligned}
$$

$$
\begin{aligned}
& \operatorname{Min} V_{t l k}(\omega) \cdot Z_{t l k} \leq V_{t l k}(\omega) \leq \operatorname{Max} V_{t l k}(\omega) \cdot Z_{t l k} \\
& \forall t \in T, \quad \forall l \in L, \quad \forall k \in L \backslash\{l\}, \quad \forall \omega \in \Omega \\
& \sum_{l \in L} \mathrm{EM}_{l k} \cdot V_{t l k}(\omega)=E_{t l k}(\omega) \quad \forall t \in T, \\
& \forall l \in L, \quad \forall k \in L \backslash\{l\}, \quad \forall \omega \in \Omega \\
& O E_{t l k}(\omega) \geq E_{t l k}(\omega)-\operatorname{Max} E \cdot Z_{t l k} \quad \forall t \in T, \\
& \forall l \in L, \quad \forall k \in L \backslash\{l\}, \quad \forall \omega \in \Omega \\
& \mathrm{UE}_{t l k}(\omega) \geq \operatorname{Min} E \cdot Z_{t l k}-E_{t l k}(\omega) \quad \forall t \in T, \\
& \forall l \in L, \quad \forall k \in L \backslash\{l\}, \quad \forall \omega \in \Omega \\
& \mathrm{OE}_{t l k}(\omega), \quad \mathrm{UE}_{t l k}(\omega), \quad V_{t l k}(\omega) \geq \quad \forall t \in T, \\
& \forall l \in L, \quad \forall k \in L \backslash\{l\}, \quad \forall \omega \in \Omega
\end{aligned}
$$

Equation (5) is the objective function to be minimized including the total distribution costs of distribution across the tactical stage, in addition to the expectation of the second-stage (recourse value) problem across the operational stage. Constraints (6) and (7) ensure that each location [except the depot] is visited exactly once in each time period. Furthermore, constraints (8), (9) and (10) generate the optimal route sequencing. Moreover, constraint (11) controls the delivering capacity, while constraint (12) ensures that enough vehicles are sent out of the depot. Constraint (13) sets the binary restrictions for the first-stage binary variables, and constraint (14) is the non-negativity constraint for all first-stage continuous variables.

The second-stage objective function (15) minimizes the total costs associated speed and their related emissions, penalties for overage and underage emissions. Constraint (16) ensures that the chosen velocity at each route in each time period at each scenario is between the realized minimum and maximum velocities on that route. Constraint (17) calculates the total emissions level of each chosen route. Constraints (18) and (19) calculate the overage or underage emissions, respectively, based on the comparison between the actual emissions that would result due to the chosen velocity and the standard emissions limits. Constraint (20) is the non-negativity constraint for the secondstage variables.

\subsection{Case studies}

In order to check the applicability of the developed model in practice as identified in Sect. 3.2, a qualitative approach has been used based on a case study analysis. The mathematical model is tested in various scenarios of real-world case studies from batch process industry. The case studies selection approach has been motivated based on the information-oriented process [8]. In this approach, the information content should be withdrawn into two phases: preliminary set of companies' selection and participation willingness in research.

In the first phase, online database containing information about multinational companies was used. The selection of the initial set of companies was based on the following specific criteria: (a) producing in batches, (b) providing different in the range of batches, (c) foster a vocation to social responsibility and environmental-friendly management, (d) involved in previous research projects in order to facilitate the information flow and (e) their data follow a regular trend, except during the holidays. Thus, sample of those data can be generalized to represent the batch process industry. A list of nine companies was selected as possible cases due to these criteria.

In the second phase, an enquiry was conducted to secure the acceptance of companies to participate in this research and to check the above listed criteria. Out of five companies agreed to be involved in the case study investigation, only two case studies from batch process industry are suitable for this research.

The first case study was conducted in one of the world's leading companies in the field of foods and beverages processing industry with employs more than 1500 employees. This company is a single huge plant with seven departments. Currently, the core business of the company includes processing and distribution of wide range of dairy and frozen products packaged under more than 40 brand names. Across 62 countries around the world, these products are ordered in batches. In 2005, the company was determined to be one step ahead on social responsibility and environmental-friendly management issues. It started by using new materials during the production processes, importing eco-efficiency methods and increasing employee involvement. Recently, the management has focused on developing solutions which consider the environmental impact of their distribution activities particularly in the field of reducing emission-related issues.

The second case study was conducted in a multinational consumer goods company and manufacturer of product ranges including personal care, household cleaning, laundry detergents, prescription drugs and disposable nappies. 
In 2014 , it recorded $\$ 83.1$ billion in sales as profit from more than 80 brands across more than 70 countries all over the world. Since 1999, the company has provided products of superior quality and value that improve the lives of the world's consumers. Their sustainability objective is to create industry-leading value with consumer-preferred brands and products while conserving resources, protecting the environment and improving the social conditions. By 2010 , the company has reduced the total $\mathrm{CO}_{2}$ emissions by about $14 \%$ per unit of production. Currently, they focus to make progress by optimizing the distribution routes and involving economic, environmental and social sustainability objectives during the planning and distribution processes.

In both case studies, demand and supply follow a regular trend. Based on the customers' demands, the number of required delivery vehicles is calculated. The current distribution strategy is to ship all the requirements of the target customer using homogeneous trucks, single-size vehicles and single-delivery shipment. All batches are packaged in a standard load pack before distribution. The unit load stacked at each pallet is $7 \mathrm{~kg}$. Therefore, the amount of each product carried in a pallet varies from one product type to another. This amount differs depending on the batch type, dimensions and size. Each vehicle is a twenty-foot equivalent unit. The numbers of pallets that can fit on a vehicle are 11. For the used heavy duty trucks which emit four times more than passenger cars on average [11], GHG emissions values are $1.726 \mathrm{~kg} \mathrm{CO}_{2}$ per vehiclemile, $0.021 \mathrm{~g} \mathrm{CH}_{4}$ per vehicle-mile and $0.017 \mathrm{~g} \mathrm{~N}_{2} \mathrm{O}$ per vehicle-mile at the average velocity $(50 \mathrm{mph}$ ) (Climate Leaders GHG Inventory [4]). These values demonstrate that $\mathrm{CO}_{2}$ represents more than $99 \%$ by mass of all the gaseous components of exhaust. Based on these values, the emission factor $\left(B_{l}\right)$ is estimated for each case study.

\section{Implementation, results and discussions}

In this section, we will discuss results obtained from the implementation of the mathematical model in the case studies. Furthermore, the quality of the stochastic programming solution will be compared to those obtained using a deterministic approach. Then, the decision of the delivery velocities and related $\mathrm{CO}_{2}$ emissions as environmental parameter will be numerically analysed.

In the first case study, the model was implemented in the liquid department. The selection of this department was based on its good reputation with about $30 \%$ of the market share. Within that department, three types of liquids are produced: long-life processed milk, flavoured milk and long-life juices. Each of these liquids is packaged under different brand names with different volumes.
The second case study was carried out in the beauty, hair and personal care liquids section. The developed model was implemented in the shampoo department (of the liquids section) which brought the company about $8 \%$ of its income.

All the required characteristics which are related to demand, routes, depot, distribution nodes and vehicles were collected. These characteristics generate the inputs to the mathematical model as shown in Fig. 2 and Table 1. Figure 2 shows that the geographical field (to scale) of each case study represents the distance between all pairs of nodes including the depot (manufacturing facility). All the possible connections between nodes have been taken into consideration while generating the distribution network. Any node can be served directly from the depot or after another delivery points according to the optimum routes and vehicle capacity. Moreover, speed limits of these routes were noted and used to calculate the emission-related values.

The model is solved using the standard sequential linear programming. As the model class is an integer linear programming (ILP), the global optimum solution is stated using branch and bound algorithm (i.e. the solver type is branch and bound). The model is linear in both the first and second stages. All computations were run using the LINGO $^{\circledR} 10.0$ optimization package on a machine with $\mathrm{CORE}^{\mathrm{TM}}$ i5 $2.6 \mathrm{GHz}$ processor and $4 \mathrm{~GB}$ RAM under Windows 7 Enterprise.

For the distribution decisions, distribution plans were generated. These plans were then presented in the form of the optimal delivery routes. These routes should be driven to distribute the production quantities to the different locations according to customers' needs. The values of the binary decision variable $Z_{t l k}$ yielded these distribution routes. The results of the second stage were also optimized. The values of the optimum velocity and its related emission quantities were determined. These values are yielded only when the route is selected-when the vehicles' routing decision variable value $Z_{t l k}$ equals one. The main results are summarized in Table 2.

After obtaining the optimal solution, the analysis of the outcomes is performed to show the importance of modelling the presented framework in this way. This analysis involves: (a) evaluating the usefulness of the stochastic modelling approach instead of a deterministic one and (b) sensitivity analysis of the velocity because it is the most influential model parameter around the optimal values.

Commonly, due to the computational difficulty of solving the stochastic models, the usefulness of the stochastic modelling approach is always questionable. To check the suitability of using stochastic programming, the solutions of the stochastic programming model to that of a 


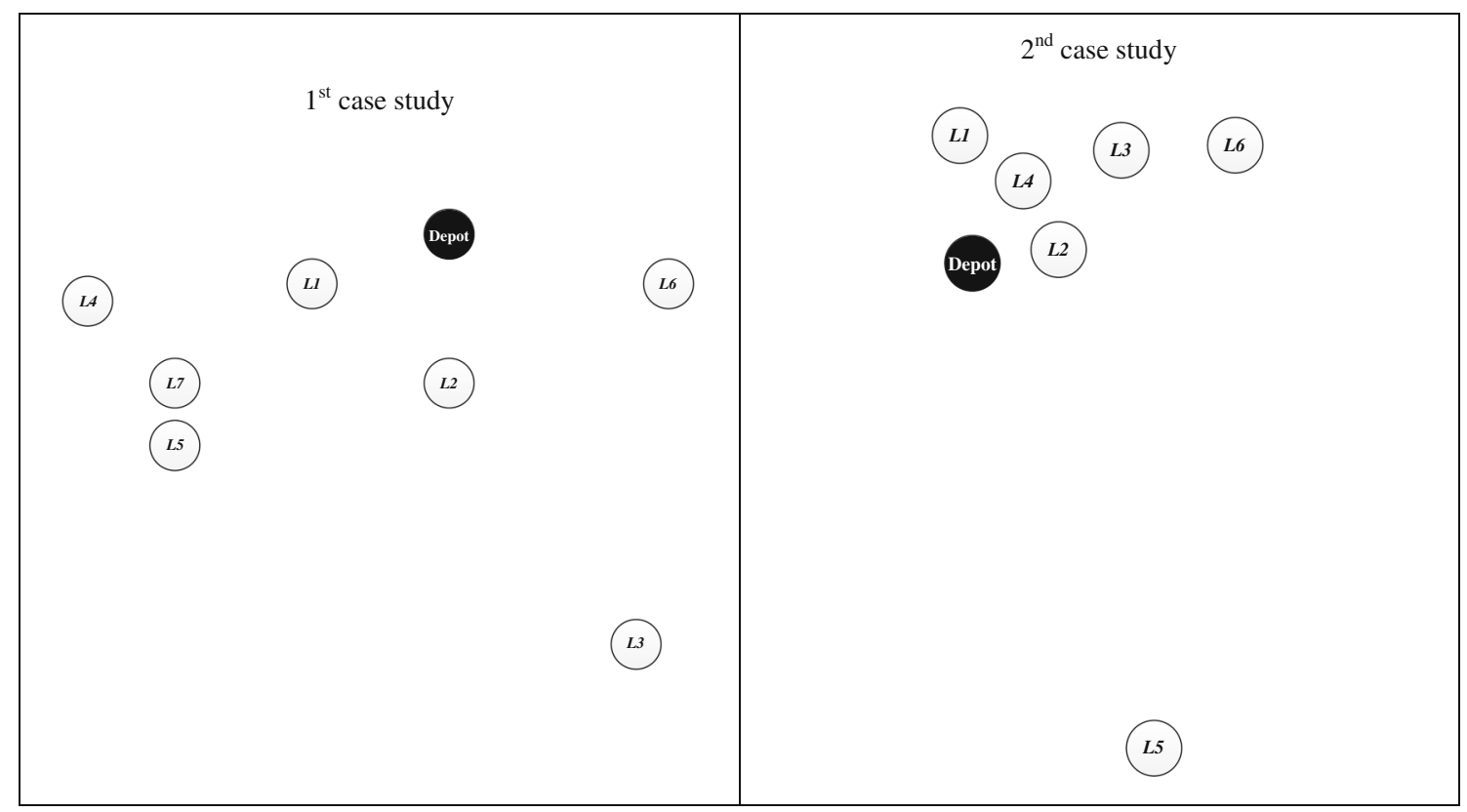

Fig. 2 Geographical field of case studies to scale

Table 1 Case study characteristics

\begin{tabular}{lll}
\hline Character & First case study & Second case study \\
\hline Weekly demand (batches) & 14,505 & 16,6215 \\
Time periods & 2 & 3 \\
Number of batches families & 7 & 10 \\
Nodes (including depot) & 8 & 7 \\
\hline
\end{tabular}

deterministic optimization problem are quantified. For stochastic models, when multiple runs of the models are averaged out, it still cannot be concluded that the output will also be the same. But after looking at the definition of the models and how the results are produced, it is possible to get a better understanding of how models will behave under different inputs and different scenarios [24].

In order to obtain the deterministic values of the developed model, the stochastic values of the delivery velocities and their related emission values are replaced by their average values. Therefore, the environmental objective function and constraints will be reformulated as follows:

$$
\begin{aligned}
& \min \sum_{t \in T} \sum_{p \in P} \mathrm{DC}_{l k} \cdot D_{l k} \cdot Z_{t l k}+\sum_{l \in L} \sum_{k \in L \backslash\{l\}} \sum_{t \in T}\left[\left(\mathrm{EM}_{l k} \cdot B_{l}\right)\right. \\
& \left.\cdot V_{0 t l k}+F_{l k} \cdot \mathrm{OE}_{t l k}+G_{l k} \cdot \mathrm{UE}_{t l k}\right] \\
& \operatorname{Min} V_{t l k} \cdot Z_{t l k} \leq V_{0 t l k} \leq \mathrm{Max} V_{t l k} \cdot Z_{t l k} \quad \forall t \in T, \quad \forall l \in L, \quad \forall k \in L \backslash\{l\} \\
& \sum_{l \in L} \mathrm{EM}_{l k} \cdot V_{0 t l k}=E_{t l k} \quad \forall t \in T, \quad \forall l \in L, \quad \forall k \in L \backslash\{l\}
\end{aligned}
$$

$\mathrm{OE}_{t l k} \geq E_{t l k}-\operatorname{Max} E \cdot Z_{t l k} \quad \forall t \in T, \quad \forall l \in L$, $\forall k \in L \backslash\{l\}$

$\mathrm{UE}_{t l k} \geq \operatorname{Min} E \cdot Z_{t l k}-E_{t l k} \quad \forall t \in T, \quad \forall l \in L$, $\forall k \in L \backslash\{l\}$

$\mathrm{OE}_{t l k}, \mathrm{UE}_{t l k} \geq 0 \quad \forall t \in T, \quad \forall l \in L, \quad \forall k \in L \backslash\{l\}$

Furthermore, two values should be calculated: the expected recourse problem (RP) solution value and the expected result of using the expected value (EEV). RP is the generated value when a model is solved using the real values. EEV corresponds to one particular scenario in
Table 2 Main computational results

\begin{tabular}{lll}
\hline Character & First case study & Second case study \\
\hline Total variables (integers) & $10,702(177)$ & $18,413(229)$ \\
Constraints & 13,042 & 22,408 \\
Number of iterations till optimality & 945,891 & 15,065 \\
Objective function value $€$ & $288,508.6$ & $460,304.3$ \\
Total number of generated routes & 5 & 16 \\
\hline
\end{tabular}


Table 3 VSS results for different tested scenarios

\begin{tabular}{lllllr}
\hline & Number of scenarios & RP value $€$ & EEV value $€$ & VSS value $€$ & (VSS/RP) \% \\
\hline First case study & 10 & 287,292 & 324,797 & 37,506 & 13.055 \\
& 20 & 288,509 & 320,230 & 31,722 & 10.995 \\
& 30 & 289,202 & 315,840 & 26,638 & 9.211 \\
\multirow{5}{*}{ Second case study } & 40 & 289,287 & 307,590 & 18,303 & 6.327 \\
& 50 & 289,590 & 304,341 & 14,751 & 5.093 \\
& 10 & 460,577 & 508,680 & 48,103 & 10.444 \\
& 30 & 460,304 & 499,780 & 39,476 & 8.576 \\
& 40 & 460,453 & 495,655 & 35,202 & 7.645 \\
& 50 & 459,925 & 484,368 & 24,444 & 5.315 \\
\end{tabular}

which the corresponding expected value $(\mathrm{EV})$ replaced the observation of the random values. The difference between the RP and EEV is defined as the value of the stochastic solution (VSS). The VSS is the concept that precisely measures the quality of a decision [1].

To display these values, datasets of 10, 20, 30, 40 and 50 scenarios were tested. Table 3 shows the values of running these numbers of scenarios and their corresponding running time. For each dataset, the RP, EEV and VSS values are calculated. Moreover, the VSS as a percentage of the overall RP solution is reported. Since all VSS have positive values, dealing with uncertainty really matters. Therefore, optimal solutions are sensitive to the value of the random elements. The difference between the optimal solution (generated from the stochastic modelling) and the expected solution (generated form the deterministic modelling) is between 13 and 4 percentages for datasets of 10, 20, 30, 40 and 50 scenarios. The VSS values verify the significance and benefits of using the stochastic modelling approach. In the deterministic model, the average delivery velocity $(\mathrm{km} / \mathrm{h})$ was 75 and the average emission factor $\left(\mathrm{kg} \mathrm{CO}_{2} / \mathrm{km} / \mathrm{h}\right)$ was 1.2 in both case studies.

Moreover, the delivery velocity of the base in the second stage in the developed stochastic model is examined. The generated emissions during distribution depend mainly on the delivery velocity as mentioned earlier. The optimum transporting velocity values were generated in the case studies. When the required batches are delivered faster than the optimum, more emissions are generated which is directly proportional to emission costs. Table 4 shows the effect of changing the delivery velocities in the distribution phases on the total cost.

Results show that the change of the velocity negatively or positively results in the increase in the value of the total cost. However, decreasing the velocity parameter increases the costs at a higher rate more than the effect of increasing this factor. The optimal solution guarantees that the output schedule generated with the least cost value. The conclusion is that the optimum solution is more sensitive to decreasing the delivery velocity time than increasing it.
Table 4 Change of objective cost value in response to change of velocity

\begin{tabular}{llll}
\hline $\begin{array}{l}\text { Velocity } \\
\text { changing } \\
(\%)\end{array}$ & $\begin{array}{l}\text { \% of the } \\
\text { environmental/total } \\
\text { cost }\end{array}$ & $\begin{array}{l}\text { Sensitivity } \\
\text { coefficient in the } \\
\text { environmental } \\
\text { cost }\end{array}$ & $\begin{array}{l}\text { Sensitivity } \\
\text { coefficient in } \\
\text { the total cost }\end{array}$ \\
\hline-25 & 32.07 & 1.35 & 0.43 \\
-20 & 32.02 & 1.08 & 0.34 \\
-15 & 31.96 & 0.81 & 0.25 \\
-10 & 31.90 & 0.54 & 0.17 \\
-5 & 31.84 & 0.27 & 0.08 \\
0 & 31.78 & 0.00 & 0.00 \\
5 & 31.84 & 0.24 & 0.07 \\
10 & 31.89 & 0.49 & 0.15 \\
15 & 31.94 & 0.74 & 0.23 \\
20 & 32.00 & 0.98 & 0.31 \\
25 & 32.05 & 1.23 & 0.39 \\
\hline
\end{tabular}

The total cost surges when the velocities fall due to rise in the lateness penalty. On the other hand, when the velocities increase, the total cost escalates due to heightened level of the emission penalty.

In sum, the results of the model analysis show that it can generate the optimal solution for the batch process industry. This is verified and successfully implemented in two real-life industrial case studies that prove the model's capability as a decision support system which addresses both routing and scheduling distribution decisions. Using the previous scenarios, the flexibility of the proposed integrated green model is proved while taking into consideration most of the special features in the batch process industry.

\section{Conclusions}

The main goal of this study is to show the potential of the introduction of a green logistics model in batch process industry. The proposed model can be considered a decision 
support tool for distribution planning. It helps the decisionmaker in delivering decisions on both tactical and operational levels. The advantage of using the delivery velocities values and the related emissions has been proved.

On the one hand, the importance of optimizing transport planning was shown in cost and emission savings. Based on the results, a batch process facility improves its efficiency objectives from economic and ecological perspectives. On the other hand, the optimal transporting velocity ranges should be strictly observed during logistics physical activities. Due to the uncertain dynamic environment, controlling delivery velocities is not that easy in practice. For these reasons, extra costs must be incurred in training and education of human resources who are involved in the distribution processes in order to increase their level of green awareness and knowledge. This could be studied in depth for further research.

Furthermore, the proposed model supports academic green logistics models and real-world supply chain decision making in batch process industry. Developing and implementing the proposed green logistics model provide a practical tool which links being green and being economically successful. Within the globalized market, greening the batch supply chain through an environmentally friendly logistics network design guarantees competitive advantages and meets the customers' expectations and social responsibilities requirements.

In addition, the model can also be applied to other companies in order to assess the accuracy of the conclusions reached in this paper. Further research may also lead to finding cleaner routes, through the development of exact or approximate algorithms. Additionally, adding other sources of uncertainties within the distribution phases will positively enrich the credibility of this model. In this dynamic environment, uncertainties are represented by the gap between the planned and actual system status. This gap shows a new challenge for the management of complex and dynamics supply chain processes. Finally, defining a useable way to obtain real measures for $\mathrm{CO}_{2}$ emissions will highly improve the accuracy of the results and therefore the decision of the driver during delivery.

There are many prospects for further research on this work. One is involving the green aspects in the production stage, rather than just in the distribution phase. A second addition could be using different emission estimation ways and comparing them altogether. A third extension is adding other sources of uncertainty, such as the production costs and capacities. A fourth and final direction could be calculating the effect of considering the social criteria such as improving labour conditions and human rights.

Acknowledgments The authors appreciate the support of Ms. Yosra Abdelkhalek, Product Supply Specialist, Mondelēz International
(Kraft Foods), and Mr. Eslam Saeed Mekky, Supply Network Operations Manager, Procter \& Gamble, for providing us the case study data. We would also like to thank Dr. Aly Megahed, IBM ResearchAlmaden Research Center-for his valuable technical support, help and advice. Special thanks to Professor Yahaya Yusuf, University of Central Lancashir, for his academic support. This research was financially supported by the Deutscher Akademischer Austausch Dienst (DAAD) and the Egyptian Ministry of Higher Education under Grant GERLS 2010. The authors would like to thank International Graduate School for Dynamics in Logistics (IGS) in University of Bremen and the anonymous reviewers.

Funding This study was funded by the Deutscher Akademischer Austausch Dienst (DAAD) and the Egyptian Ministry of Higher Education under Grant GERLS 2010.

\section{Compliance with ethical standards}

Conflict of interest The authors declare that they have no conflict of interest.

Open Access This article is distributed under the terms of the Creative Commons Attribution 4.0 International License (http://crea tivecommons.org/licenses/by/4.0/), which permits unrestricted use, distribution, and reproduction in any medium, provided you give appropriate credit to the original author(s) and the source, provide a link to the Creative Commons license, and indicate if changes were made.

\section{References}

1. Birge JR, Louveaux F (2011) Introduction to stochastic programming, 2nd edn. Berlin, Springer

2. Buehler R, Pucher J (2011) Sustainable transport in Freiburg: lessons from Germany's environmental capital. Int J Sustain Transp 5:43-70

3. Cari T, Gold H (2008) Vehicle routing problem. ISBN 978-9537619-09-1, InTech

4. Climate Leaders GHG Inventory Protocol (2008) Optional emission from commuting, travel, and product transport. Office of air and radiation. United State Environmental Protection Agency. http://www.epa.gov/climateleadership/. Accessed 03 Aug 2015

5. Dekker R, Bloemhof J, Mallidis I (2012) Operations research for green logistics-an overview of aspects, issues, contributions and challenges. Eur J Oper Res 219(3):671-679

6. Demir E, Bektaş T, Laporte G (2014) Invited review-a review of recent research on green road freight transportation. Eur J Oper Res 237(3):775-793

7. Eglese R, Bektaş T (2014) Vehicle routing: problems, methods, and applications; green vehicle routing. Society of industrial and applied mathematics: MOS-SIAM series on optimization, 2nd edn, $437-458$

8. Flyvbjerg B (2001) Making social science matter. Cambridge University Press, Cambridge

9. Gao S, Qi L, Lei L (2015) Integrated batch production and distribution scheduling with limited vehicle capacity. Int J Prod Econ 160:13-25

10. Hall NG, Potts CN (2005) The coordination of scheduling and batch deliveries. Annu Oper Res 135(1-4):41-64

11. Kean AJ, Harley R, Kendal G (2003) Effects of vehicle speed and engine load on motor vehicle emissions. Environ Sci Technol 37(17):3739-3746 
12. Kopfer HW, Schönberger J, Kopfer H (2014) Reducing greenhouse gas emissions of a heterogeneous vehicle fleet. Flex Serv Manuf J 26(1-2):221-248

13. Kumar S, Teichman S, Timpernagel T (2012) A green supply chain is a requirement for profitability. Int $\mathbf{J}$ Prod Res 50(5):1278-1296

14. Maden W, Eglese RW, Black D (2010) Vehicle routing and scheduling with time-varying data: a case study. J Oper Res Soc 61:515-522

15. Min H, Kim I (2012) Green supply chain research: past, present, and future. Logist Res 1(4):39-47

16. Palanivelu P (2010) Green logistics. White paper. TATA Consultancy Services Limited (TCS), pp 1-20

17. Park YB (2005) An integrated approach for production and distribution planning in supply chain management. Int $\mathrm{J}$ Prod Res 43(6):1205-1224

18. Pishvaee MS, Jolai F, Razmi J (2009) Technical paper: a stochastic optimization model for integrated forward/reverse logistics network design. J Manuf Syst 28(4):107-114
19. Pishvaee MS, Torabi SA, Razmi J (2012) Credibility-based fuzzy mathematical programming model for green logistics design under uncertainty. Comput Ind Eng 62:624-632

20. Qian J, Eglese R (2016) Fuel emissions optimization in vehicle routing problems with time-varying speeds. Eur J Oper Res 248(3):840-848

21. Srinivasu R (2014) Fast moving consumer goods retail market, growth prospect, market overview and food inflation in Indian market-an overview. Int $\mathbf{J}$ Innov Res Sci Eng Technol 3(1):8422-8430

22. Srivastava SK (2007) Green supply-chain management: a stateof-the-art literature review. Int J Manag Rev 9(1):53-80

23. Ubeda S, Arcelus FJ, Faulin J (2011) Green logistics at Eroski: a case study. Int J Prod Econ 131:44-51

24. Van Dam KH, Adhitya A, Srinivasan R, Lukszo Z (2009) Critical evaluation of paradigms for modelling integrated supply chains. Comput Chem Eng 33(10):1711-1726 